\title{
Application of Image Processing in Neurobiology: Detection of Low Signals with High Spatial Resolution and a Non-Uniform Variance
}

\author{
Yasmina CHITTI \\ Unité de Neurocybernétique Cellulaire \\ 280, Bd Ste Marguerite \\ 13009 Marseille (FRANCE) \\ Tel: (33) 91750200 Fax: (33) 91262038 \\ email: chitti@cptsu2.univ-mrs.fr
}

\begin{abstract}
Optical imaging of neuronal activity is a recent method for studying information processing by neuronal networks. It is known that neuronal activity was generated by the activity of membrane proteins, called "ion channels".

To see the spatial action of channels on a single neuron, we use an imaging system combining a CCD camera mounted on an inverted microscope and fluorescence voltage sensitive probe and taking images of fluorescence intensity with high spatial resolution and low noise.

To visualize the difference between an excitated and a quiet state of a neuron we must compute the relative variation in fluorescence between both images representing both states. We obtained a very noisy resulting image of low signals. We have shown that its variance is non uniform and in inverse ratio to the square of raw data intensity and that the highest variances are located on the non-biological background.

We have developed a nonlinear filtering based on local image segmentation. The algorithm filters all the noise in the non-biological background and outlines the shape of the cell body. Tests of significance between groups of intensities have shown that the response of the neuron is patchy and biological experiments show that the patchyness is bound to electrical activities of the neuron.
\end{abstract}

\section{Introduction}

Several methods allows neurobiologists to study neurons. The most often used consists in introducing a microelectrode into a cell body of a single neuron. The electrode stimulates the neuron with an electrical impulse. The same microelectrode records the electrical response of the neuron, called action potential. It is known that the action potential was generated by the activity of membrane proteins, called "ion channels" but a single microelectrode can only measure the spatial sum of the potential fields created from each active channel of the whole membrane [5]. 
Optical recording with photodiode devices and voltage sensitive dyes have been previously used to detect changes in transmembrane voltage at high temporal resolution [4]. Bath-applied dye binds to the external membrane and acts as a molecular transducer that transforms changes in membrane potential into optical signals. It has been shown that the fluorescence of the stained neuron correlates linearly with its electrical activity. Taking advantage of both the linearity and the microsecond response time of the dye, the fluorescence changes in the neuronal membrane during recorded individual action potentials can be imaged. During the exposure time, the rapid transmembrane voltage changes produce local changes in the emitted fluorescence which are stored in the image. This image is the spatial counterpart of the time information conveyed by a microelectrode recording.

The mean diameter of a single cultured neuron is about $30 \mu \mathrm{m}$. The sources of action potential are about $1 \mu \mathrm{m}$ large and the emitted signal is very low. Neurobiologists and physicists have cooperated to work out a high spatial resolution and low noise imaging system and an adequate image processing.

\section{Optical Recording System}

In order to check the response of the stained neuron, a microelectrode is introduced in its membrane. The electrode stimulates the neuron and records the action potential. The emission spectra of the probe linearly shifts with changes in membrane potential. A linear optical filter transforms the shift into changes in fluorescence intensity. Images of fluorescence are taken with a liquid nitrogen cooled charge-coupled device (CCD) camera mounted on an inverted microscope. The imaging system is a low noise ( $<10$ electrons r.m.s) system and it allows us to take $189 \times 70$ pixels images with a $1 \mu \mathrm{m}^{2}$ spatial resolution. Figure $1, \mathrm{~A}$ shows an image of the fluorescent neuron obtained with a such imaging system.

We want to compare two excitation states of a neuron: at rest -without stimulation- and during an action potential -after a stimulation. The changes in potential between this two excitation states is around $100 \mathrm{mV}$ and correspond to a shift of the emission spectra of the probe around five percent of its initial value and a variation of fluorescence intensity around three percents: the variation does not allows us to see any difference between two images corresponding to the both states of excitation of the neuron.

\section{$3 \quad$ Image Processing}

In order to visualize the difference between the both studied states and to see the spatial distribution of active channels, it is necessary to compute the relative variation in fluorescence between the both images. Experimental noises like bleaching (the intensity of fluorescence decreases with the lighting of the field), cellular movements (images must be quickly taken)... have been estimated and removed. However, the result of the computation is very noisy as can be seen 
in Fig. 1,B. When the neuron is not stimulated the variation of fluorescence intensity of the cell is around $0 \%$ but the non-biological background is very noisy. When the neuron is stimulated we can see the edge of the cell and observe that the noise is higher on the background than on the cell.

As the noise provided by CCD is a Poisson-noise, the variance of every pixel intensity of the image of the fluorescent neuron is proportional to the pixel intensity and has a different value in each $(x, y)$ pixel [1]. The study of the variance resulting of the relative variation computation shows that the variance is non uniform:

$$
V(x, y)=V\left(\frac{f(x, y)-g(x, y)}{f(x, y)}\right)=\frac{g^{2}(x, y)}{f^{4}(x, y)} * \sigma_{1}^{2}(x, y)+\frac{1}{f^{2}(x, y)} * \sigma_{2}^{2}(x, y)
$$

where

1. $f(x, y)$ is the spatial intensity of the $(x, y)$ pixel of a control image of the neuron at rest.

2. $g(x, y)$ is the spatial intensity of the $(x, y)$ pixel of a test image where the neuron was either stimulated or at rest.

3. $\sigma_{1}^{2}(x, y)=c * f(x, y)$ and $\sigma_{2}^{2}(x, y)=c * g(x, y)$ are their variance.

The computation give us a chart of the spatial variance (showed in Fig. 1,C):

$$
V(x, y)=c * \frac{g(x, y)}{f^{2}(x, y)} *\left[1+\frac{g(x, y)}{f(x, y)}\right]
$$

The variance of the relative variation is in inverse ratio to the raw data intensity. In fact, as spots of high intensity of fluorescence in original images are located on biological membrane, the variance of the result image of relative variation is higher on the non-biological background than on biological membranes. The intensities of two pixels next to each other of the non-biological background of the raw image of fluorescence are similar but the intensities of these both pixels in the relative variation image really differ. Only a nonlinear processing based on local features will be able to filter this image.

The developed algorithm is a nonlinear filter based on local image segmentation[3]. We compute the variation between the intensity of each pixel and the mean intensity of the 4-neighbouring pixels. If the variation is greater than a local threshold which is proportionnal to the intensity of the centered pixel, we removed the centered pixel else the pixel is not removed. As Fig. 1,D shows, the algorithm filters all the noise in the non-biological background and outlines the shape of the cell body.

To localize activity areas, the result was filtered with a $3 \times 3$ median filter as Fig. 1,E presents. After the filtering by dynamic thresholding segmentation and by a median filter, active areas appear on the cell when the neuron was excited and do not appear when the neuron is at rest. 


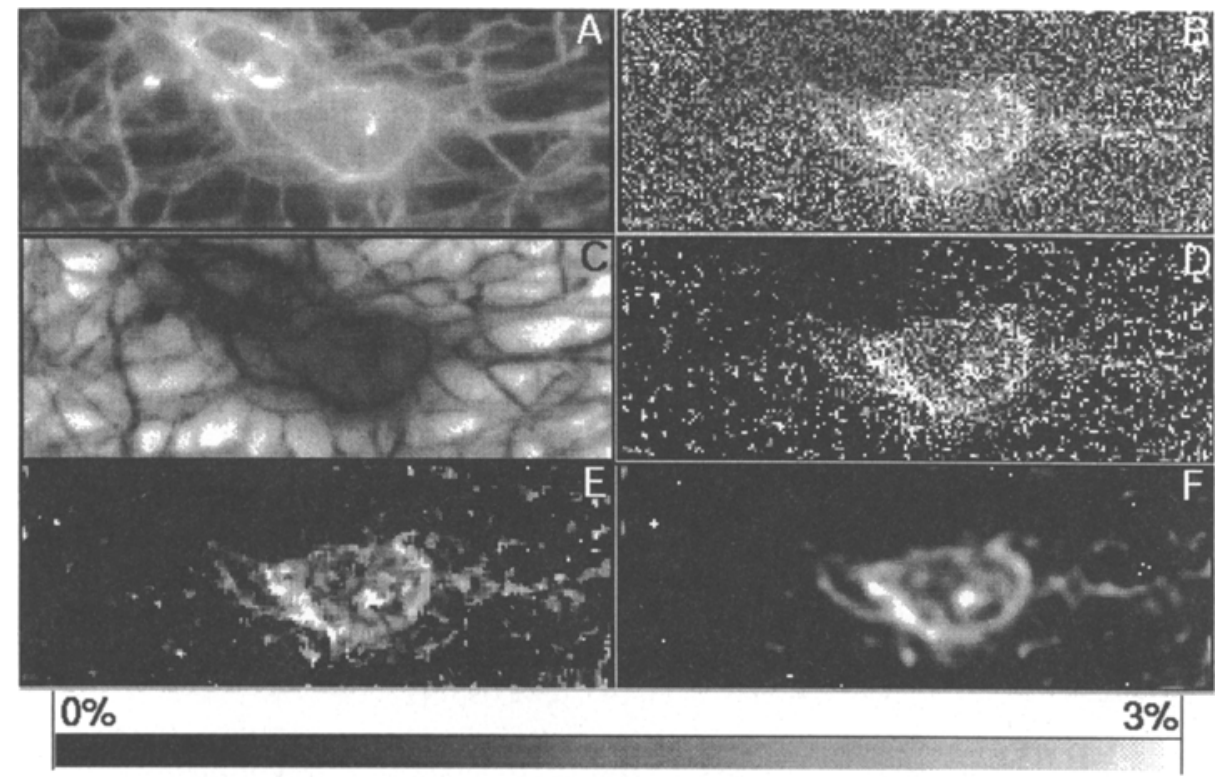

Fig. 1. Detection of active channels on a neuron membrane.

(A) Image in fluorescence after incorporation of the dye (RH237). (B) Relative variation of fluorescence $(d F / F)$ between an image of the neuron at rest and an image of the excited neuron. (C) Map of local variances of the $\mathrm{C}$ image. (D) Filtering of image $B$ by local segmentation removing the noise of the non-biological part of the image.

(E) Filtering of the image D by a $3 \times 3$ median filter removing the spot of noise.

(F) Detection of active channels by using the wavelet transform.(Scale used for D-F images.)

\section{Results}

The significance of the result was tested by several ways.

1. The first test consists to compare the mean intensity of two areas which have almost the same intensities. If two samples, A and B, are extracted, the result of the test give us the confidence of $p \%$ with which mean $(A)$ and $\operatorname{mean}(B)$ differ.

Generally, the intensity after filtering range from $0 \%$ to $2 \%$ of variation and four samples of $0.5 \%$ large can be distinguished.

2. An experimental model which contains all experimental evaluated noises was evolved, filtered and tested with the previous test of significance. The same significance is obtained for the both experimental cases. These results show that the patchy response of the neuron is not due to the non uniform spatial distribution of fluorescence but due to differences in the emission wavelength of the probe and so to differences in local potential. 
3. To increase the spatial resolution, images of relative variation will be filtered in Wavelets space with the à trous algorithm [6] by taking in account the spatial distribution of the variance. The evaluation of the intensity, the location and the size of each active areas on the membrane will be better evaluated.

Many biological experiments prove that the detected active channels are bound to the electrical activity of the membrane of the neuron. We have developped a new method to see the electrical excitation of a cultured neuron which add a new dimension to electrical reccordings of neurons [2].

\section{References}

1. F.J. Anscombe: The Transformation of Poisson, binomial and negative-binomial data. Biometrika 15,246-254 (1948)

2. P. Gogan, I. Schmiedel-Jakob, Y. Chitti and S. Tyč-Dumont: Fluorescence Imaging of Local Electric Fields During Excitation of Single Neurons in Culture. Biophys. J. (accepted)

3. R.C. Gonzalez and P. Wintz: Digital Image Processing. Addison Wesley 1987.

4. A Grinvald, R. Hildesheim, I.C. Farber, and L. Anglister: Improved Fluorescent Probes for the Measurement of Rapid Changes in Membrane Potential. Biophys. J. 39,301-308 (1982)

5. C. Hammond and D. Tritsch: Neurobiologie Cellulaire I. Doin 1990.

6. M. Holdschneider, R. Kroland-Martinet, J. Morlet, and P. Tchamitchian: A realtime Algorithm for Signal Analysis with the help of the Wavelet transform. In J.M. Combes et al.: Wavelets. Berlin: Springer 1989, pp 286-297 